\title{
Transcatheter technique now standard for Secundum ASD Closure, A case report of our first experience.
}

\author{
A Q M Reza', A B Siddique ${ }^{2}$, M S U Talukder ${ }^{3}$, S Munwar ${ }^{4}$, A H M W Islam5 , S R Shohel ${ }^{6}$, M A Ghani ${ }^{7}$
}

\begin{abstract}
Introduction: Atrial septal defect (ASD) is one of the more commonly recognized congenital cardiac anomalies presenting in adulthood. Atrial septal defect is characterized by a defect in the interatrial septum allowing pulmonary venous return from the left atrium to pass directly to the right atrium. Depending on the size of the defect, size of the shunt, and associated anomalies, this can result in a spectrum of disease from no significant cardiac sequelae to right-sided volume overload, pulmonary arterial hypertension, and even atrial arrhythmias.
\end{abstract}

Surgical closure of atrial septal defects has been practiced successfully for 50 years, nowadays reaching the expectation of zero mortality and an optimal functional result. In the attempt to minimise operative morbidity, practice has moved to a less invasive surgical approach, including minimal skin incisions, reduced opening of the thoracic cage and alternative vascular access sites. More recently, the development of catheter-based technology has advanced device occlusion as the first option for closure of ostium secundum atrial septal defects. This mode of therapy appears now to be widely accepted, based on the obvious advantage of saving the patient from open heart surgery, allowing subsequently faster rehabilitation. ${ }^{2}$ Catheter-based closure is now considered the first-line treatment strategy for secundum atrial septal defects (ASD). Although in some cases, surgery remains the only option (eg, for those with an insufficient rim to secure a device, or those with coincidental anomalous pulmonary venous return), a percutaneous approach is suitable for the vast majority of patients with secundum ASD. As skill and experience grow, defects as large as $40 \mathrm{~mm}$ in size have been successfully closed in this manner. ${ }^{3}$ We will report a case of Secundum ASD Device Closure which was first done in Apollo Hospitals Dhaka.

Case Report: This 50 years old hypertensive (newly diagnosed), diabetic (newly diagnosed) Bangladeshi lady from Chittagong with a positive family history of heart disease was referred from outside to OPD, Apollo Hospitals Dhaka as a case of Secundum ASD for further evaluation \& treatment. Since last year she was having shortness of breath and unusual tiredness. For this reason she underwent routine checkup (OGTT, ECG \& Blood pressure monitoring). ECG showed incomplete RBBB with flattening of $\mathrm{T}$ waves from V1-V6 and for this reason she was further advised for Transthoracic Echocardiogram. Echo was done on 27th May 2009 which showed ASD(Secundum) with left to right shunt about $23 \mathrm{~mm}$ approximately, Dilated RA(40mm), RV(30 $\mathrm{mm}), \mathrm{PA}(23 \mathrm{~mm})$ with mild pulmonary hypertension(PASP $55 \mathrm{mmHg}$ ) \& Mild Tricuspid Regurgitation. Pulmonary flow was $1.73 \mathrm{~m} / \mathrm{sec} \&$ PPG $12.03 \mathrm{mmHg}$.

On examination in Apollo Hospitals Dhaka, at OPD revealed
Pulse - 80 bpm regular, B.P 140/90 mmHg, Lungs - Clear, Heart - Ejection Systolic murmur with a wide fixed splitting of the second heart sound. She was started with antihypertensives \& was put on strict diabetic diet and was advised for TEE, CAG \& Cardiac catheterization. CAG and Cardiac catheterization was done on 04.06.09. CAG revealed Normal Epicardial Coronary Arteries and Cardiac catheterization revealed Qp : Qs = 2.73: 1, PVR = 5 wood unit, L-R shunt $63 \%$, significant step up of $\mathrm{O}_{2}$ saturation in mid RA and she was advised for ASD Device closure.

On 08.06.09 TEE was done which revealed Moderate size ASD (Secundum) about $25 \mathrm{~mm}$ with left to right shunt and good rim size of IAS.

On 19.07.09 ASD device closure was done successfully through right femoral approach under local anaesthesia without any sedatives and without Trans Esophageal Echo (TEE) with a $26 \mathrm{~mm}$ device. Transthoracic Echo was used per operatively \& post operatively and device was implanted with no residual shunt. Patient was discharged home on 22.07.09 with Dual Antiplatelet, antihypertensives and advice to come for a follow up 1,3 and 6 month. The followup scheme after device occlusion comprises a physical examination, routine blood (CBC), CXR and transthoracic echocardiography. Her first follow up after 1 month, patient had no complaints, Physical examination revealed Pulse-68 bpm regular, B.P 125/80 mm Hg, Lungs-Clear, HeartNormal, 1st \& 2nd heart sound, Echo was done which showed device normaly in situ with no residual shunt. At 3rd month CBC \& CXR were done which were within normal limit. CXR showed device in position. At 6 th months routine blood tests were done which showed no abnormality. Echo showed device in situ with no residual shunt and chamber dimensions and other parameters are within normal limit - RA (30), RV (27.8) and PA (19.5), Pulmonary Flow $0.99 \mathrm{~m} / \mathrm{sec}$, PPG-3.9 $\mathrm{mmHg}$, PASP $29 \mathrm{mmHg}$. Antiplatelets were discontinued from 6th month after the procedure.

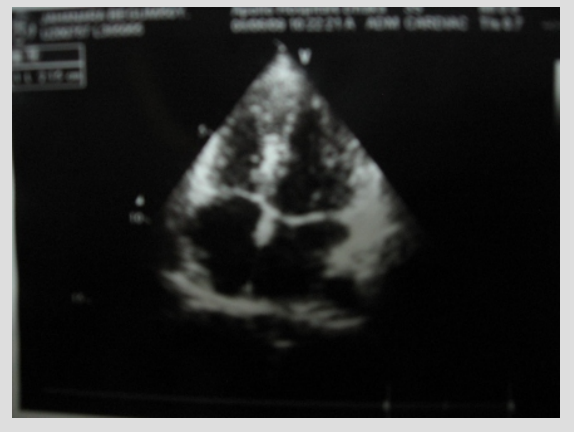

Figure 1: Apical 4 chamber view showing ASD. 


\section{Transcatheter technique}
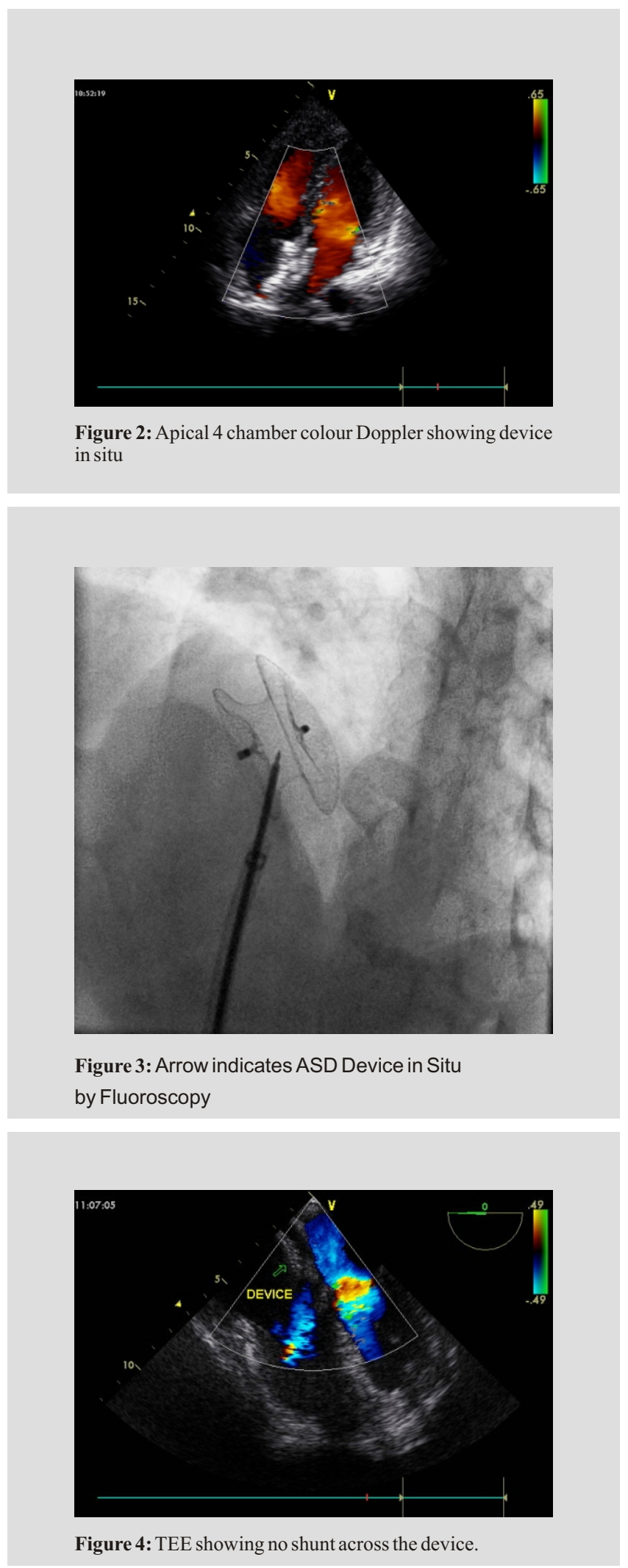

Discussion: Transcatheter closure is becoming an accepted technique for closure of patent foramen ovales and some ASDs. Because it can be performed without general anesthesia, without cardiopulmonary bypass, and without an incision, the technique must be considered an attractive alternative to conventional surgical closure. Although the cost of the device and the implantation facilities may be similar to those of surgical closure, hospitalization is shortened or eliminated and time off from work is greatly reduced. A close working relationship between the surgeon and the interventionist will permit successful and safe application of this technique. The surgeon's role continues to be that of patient advocate, recognizing favorable and unfavorable anatomy, and participating in the selection of patients for the transcatheter approach . ${ }^{4}$

In our first experience we have successfully closed a Secundum ASD in a middle aged women without any minor or major complications by Transcatheter device closure. Our achievement was, we did it without sedations and we used per procedural Transthoracic Echocardiography instead of Transesophageal Echocardiography for positioning of the device during implantation and to check whether there is any residual shunt present or not and ensured better comfort of the patient.

Conclusion: In view of the high success rate of complete closure, safety of device closure, and growing experience in use of this device, it seems likely that device closure of ASD will become the preferred mode for closure of ASD with suitable anatomy.

\section{References:}

1. Markham LW, Cribbs MG. Atrial Septal Defect. eMedicine from WebMD, Updated: Jan 21, 2010.

2. Bov Th, Fran ois K, De Groote Suys KB, De Wolf D, Nooten GV, Closure of atrial septal defects: is there still a place for surgery? Acta Chir Belg. 2005:105:497-503.

3. Sharp A, Malik I. Secundum atrial septal defects:time to close them all ? Heart. 2008;94:1120-1122.

4. Austin EH. Transcatheter closure of atrial septal defects. J Thorac Cardiovasc Surg. 2003;125:85-86. 